\title{
Stochastic Sensor Scheduling with Application to Networked Control
}

\author{
Farhad Farokhi and Karl H. Johansson
}

\begin{abstract}
We consider stochastic sensor scheduling with application to networked control systems. We model sampling instances (in a networked system) using jumps between states of a continuous-time Markov chain. We introduce a cost function for this Markov chain which is composed of terms depending on the average sampling frequencies of the subsystems and the effort needed for changing the parameters of the underlying Markov chain. By extending Brockett's recent contribution in optimal control of Markov chains, we extract an optimal scheduling policy to fairly allocate network resources (i.e., access to the network) among the control loops. We apply this scheduling policy to a networked control system composed of several scalar decoupled subsystems and compute upper bounds for their closed-loop performance. We illustrate the developed results numerically on a networked system composed of several water tanks.
\end{abstract}

\section{INTRODUCTION}

In digital control literature, many rules have been introduced for determining the sampling rates of continuous time systems [1], [2]. However, these methods mostly yield conservative results when applied to networked control systems because they often assume that the sampling is done periodically with a fixed rate. This conservatism is stemming from the fact that the subsystems are sampled with a fixed sampling rate even if they are inactive. In addition, to avoid interference between control loops, the network manager should schedule the communication instances in a networked system considering the worst-case possible scenario (i.e., the maximum number of active control loops). This becomes troublesome when dealing with ad-hoc networked control systems where at any given time, several control loops may join or leave the networked system or switch between active and inactive states. Therefore, we need a scheduling policy to set the sampling rates of the control loops adaptively according to their required closed-loop performance and the overall network resources. In this paper, we use continuous-time Markov chains to develop an optimal stochastic scheduling policy that can automatically determine the sampling rates of the subsystems in a networked system based on the number of active control loops.

Specifically, we use time instances of the jumps between states of the aforementioned continuous-time Markov chain to model the sampling instances of the subsystems. We introduce a cost function for this Markov chain which is the summation of the average sampling frequencies of the subsystems and the effort needed for changing the scheduling

The authors are with ACCESS Linnaeus Center, School of Electrical Engineering, KTH Royal Institute of Technology, Stockholm, Sweden. Emails: \{farokhi,kallej\}@ee.kth.se

The work was supported by the Swedish Research Council and the Knut and Alice Wallenberg Foundation. policy. We extend the results presented in [3] to minimize the described cost function. Doing so, we develop an optimal scheduling policy which fairly allocates time instances among the subsystems to transmit their state measurements to corresponding subcontrollers. This optimal scheduling policy works particularly well on ad-hoc networked systems because we can easily accommodate for the changes in the networked system by adding an extra state to the Markov chain whenever a new subsystem arrives or by removing an old state from the Markov chain whenever a subsystem leaves. Lastly, we study networked control when using the purposed stochastic scheduling policy. We consider networked control systems that are composed of scalar decoupled subsystems in feedback interconnection with impulsive controllers. We find an upper bound for their closed-loop performance as a function of the measurement noise statistics and the scheduling policy parameters. We generalize this result to two more practical controllers; i.e., pulse and exponential controllers. We also illustrate these results numerically on a networked control system composed of several decoupled water tanks.

Using Markov chains for stochastic sensor scheduling has been previously studied in [4]. However, in that paper, the authors considered discrete-time Markov chains to derive a numerically tractable algorithm for optimal sensor scheduling. Their algorithm uses one of the sensors at each time step while here, the continuous-time Markov chain can rest in one of its states to avoid sampling any of the sensors. In addition, the cost function in [4] was not written explicitly in terms of the Markov chain parameters and instead, it was described by the networked system performance when using a Markov chain for sampling the sensors. However, our scheduling policy results in a separation between designing the Markov chain parameters and networked system controller which enables us to describe the cost function (needed for deriving the optimal sensor scheduling policy) only in terms of the Markov chain parameters.

The rest of the paper is organized as follows. In Section II, we introduce the optimal stochastic scheduling policy and calculate its statistics. We study networked control system performance when using the proposed scheduling policy in Section III. In Section IV, we illustrate the developed results numerically on a networked system composed of several water tanks. Finally, we present the conclusions in Section V.

\section{A. Notation}

The sets of integer and real numbers are denoted by $\mathbb{Z}$ and $\mathbb{R}$, respectively. We use notations $\mathbb{O}$ and $\mathbb{E}$ to denote the sets of odd and even numbers. For any $n \in \mathbb{Z}$ and $x \in \mathbb{R}$, we 


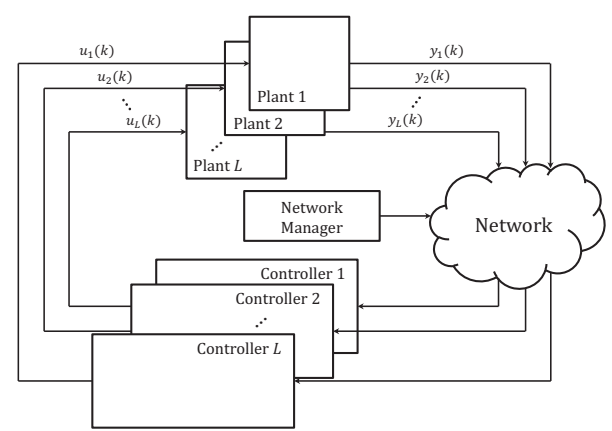

Fig. 1. Schematic diagram of the networked control system.

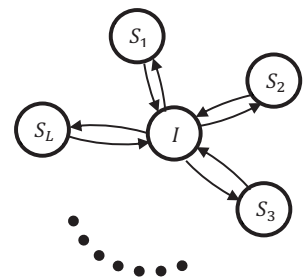

Fig. 2. Flow diagram of the continuous-time Markov chain used for modeling the stochastic scheduling policy.

define $\mathbb{Z}_{>(\geq) n}=\{m \in \mathbb{Z} \mid m>(\geq) n\}$ and $\mathbb{R}_{>(\geq) x}=\{y \in$ $\mathbb{R} \mid y>(\geq) x\}$, respectively. We use calligraphic letters, such as $\mathcal{A}$ and $\mathcal{X}$, to denote any other set.

We use capital roman letters, such as $A$ and $B$, to denote matrices. For any matrix $A, A_{i}$ denotes its $i$-th row and $a_{i j}$ denotes its entry in the $i$-th row and the $j$-th column.

Vector $e_{i}$ denotes a column vector (where its size will be defined in the text) with all entries equal zero except $i$-th entry which is equal to one. For any $x \in \mathbb{R}^{n}$, we define the entry-wise operator $x^{.2}=\left[\begin{array}{lll}x_{1}^{2} & \ldots & x_{n}^{2}\end{array}\right]^{\top}$.

\section{Stochastic Sensor Scheduling}

Let us start by modeling our stochastic scheduling policy using continuous-time Markov chains.

\section{A. Sensor Scheduling Policy}

Figure 1 illustrates the schematic diagram of a networked control system, where $L$ decoupled scalar subsystems are connected to their subcontrollers over a wireless network. A sensors in each subsystem sample its state and transmit the measurement to the corresponding subcontroller at appropriate time instances enforced by the network manager. Then, the subcontroller calculates an actuation signal and apply it directly to the subsystem. As an approximation of wireless network, we assume that the communication is instantaneous. However, we want to limit the amount of communication per time unit to reduce the energy consumption. Here, we use a continuous-time Markov chain to model the sampling instances of the subsystems. Figure 2 shows the flow diagram of this Markov chain. Every time that a jump from the idle node $I$ to a node $S_{\ell}$ occurs, we sample subsystem $\ell$ and transmit its state measurement. The idle node $I$ helps to tune the sampling frequency of the subsystems independently. We use unit vector representation [3], [5] to model this Markov chain.
We define the set $\mathcal{X}=\left\{e_{1}, e_{2}, \ldots, e_{n}\right\} \subset \mathbb{R}^{n}$ where $n=L+1$. The continuous-time Markov chain state $x(t) \in$ $\mathbb{R}^{n}$ evolves on $\mathcal{X}$, which is the reason behind naming this representation as the unit vector representation. We associate nodes $S_{1}, S_{2}, \ldots, S_{L}$, and $I$ in the Markov chain with unit vectors $e_{1}, e_{2}, \ldots, e_{L}$, and $e_{n}$, respectively. Following the same approach as in [5], we can model the Markov chain in Figure 2 by the Itô differential equation in

$$
\mathrm{d} x(t)=\sum_{\ell=1}^{L}\left(G_{\ell n}^{\prime} x(t) \mathrm{d} N_{\ell n}^{\prime}(t)+G_{n \ell}^{\prime} x(t) \mathrm{d} N_{n \ell}^{\prime}(t)\right),
$$

where $\left\{N_{n \ell}^{\prime}(t)\right\}_{t \in \mathbb{R}_{>0}}$ and $\left\{N_{\ell n}^{\prime}(t)\right\}_{t \in \mathbb{R}_{>0}}$ for $1 \leq \ell \leq L$, are Poisson counter processes with rates $\lambda_{n \ell}(t)$ and $\lambda_{\ell n}(t)$, respectively. These Poisson counters determine the rates of jump from $S_{\ell}$ to $I$ and vice versa. In addition, for $1 \leq \ell \leq L$, we have $G_{\ell n}^{\prime}=\left(e_{\ell}-e_{n}\right) e_{n}^{\top}$ and $G_{n \ell}^{\prime}=\left(e_{n}-e_{\ell}\right) e_{\ell}^{\top}$. Let us define $m=2 L$. Now, we can rearrange the Itô differential equation in (1) as

$$
\mathrm{d} x(t)=\sum_{i=1}^{m} G_{i} x(t) \mathrm{d} N_{i}(t),
$$

where $\left\{N_{i}(t)\right\}_{t \in \mathbb{R}_{\geq 0}}$ for $1 \leq i \leq m$, is a Poisson counter process with rate

$$
\mu_{i}(t)= \begin{cases}\lambda_{n,\lfloor(i-1) / 2\rfloor+1}(t), & i \in \mathbb{O}, \\ \lambda_{\lfloor(i-1) / 2\rfloor+1, n}(t), & i \in \mathbb{E},\end{cases}
$$

and

$$
G_{i}= \begin{cases}G_{n,\lfloor(i-1) / 2\rfloor+1}^{\prime}, & i \in \mathbb{O}, \\ G_{\lfloor(i-1) / 2\rfloor+1, n}^{\prime}, & i \in \mathbb{E} .\end{cases}
$$

Noting that the continuous-time Markov chain in (1) models the sampling instances $\left\{T_{i}^{\ell}\right\}_{i=0}^{\infty}$ using the jumps that occur in its state $x(t)$, we can manipulate the average sampling frequencies of different subsystems through rates $\mu_{i}(t)$. Similar to [3], we assume that we can control the rates of Poisson counters according to

$$
\mu_{i}(t)=\mu_{i, 0}+\sum_{j=1}^{m} \alpha_{i j} u_{j}(t),
$$

and thereby, control the average sampling frequencies of subsystems. Control signals $u_{j}(t)$ for all $1 \leq j \leq m$, are chosen in order to minimize the cost function

$$
J=\lim _{T \rightarrow \infty} \mathbb{E}\left\{\frac{1}{T} \int_{0}^{T} \sum_{\ell=1}^{L} \xi_{\ell} e_{n}^{\top} x(t) \mathrm{d} N_{2 \ell}(t)+u(t)^{\top} u(t) \mathrm{d} t\right\},
$$

where $\xi_{\ell} \in \mathbb{R}_{\geq 0}$ for $1 \leq \ell \leq L$, are design parameter. Note that the cost function in (6) consists of two types of terms: the term $\frac{1}{T} \int_{0}^{T} e_{n}^{\top} x(t) \mathrm{d} N_{2 \ell}(t)$ for $1 \leq \ell \leq L$, which denotes the average frequency of the jumps from $I$ to $S_{\ell}$ in the Markov chain (i.e., the average sampling frequency of subsystem $\ell$ ) and the term $\frac{1}{T} \int_{0}^{T} u(t)^{\top} u(t) \mathrm{d} t$ which penalizes the network regulation effort. If the latter term is removed, the problem would become ill-posed since the rates $\mu_{i}(t)$ (and as a result, the average sampling rates of subsystems) can be set to zero (i.e., the subsystems would work open loop with probability one). Considering 
the identity $\mathbb{E}\left\{\mathrm{d} N_{2 \ell}(t)\right\}=\mu_{2 \ell, 0}+\sum_{j=1}^{m} \alpha_{2 \ell, j} u_{j}(t) \mathrm{d} t$, we can rewrite the cost function in (6) as

$$
J=\lim _{T \rightarrow \infty} \mathbb{E}\left\{\frac{1}{T} \int_{0}^{T} c^{\top} x(t)+u(t)^{\top} S x(t)+u(t)^{\top} u(t) \mathrm{d} t\right\},
$$

where $c=e_{n} \sum_{\ell=1}^{L} \xi_{\ell} \mu_{2 \ell, 0}$ and $S \in \mathbb{R}^{m \times n}$ is a matrix whose entries are $s_{j i}=\sum_{\ell=1}^{L} \xi_{\ell} \alpha_{2 \ell, j}$ if $i=n$ and $s_{j i}=0$ otherwise. In the next subsection, through extending Brockett's recent contribution in optimal control of Markov chains [3], we find the unique minimizer of the above cost function subject to the Markov chain dynamics in (2) and the rates control law in (5). Doing so, we develop an optimal scheduling policy to fairly allocate sampling instances to the control loops in a networked system.

\section{B. Optimal Sensor Scheduling}

We start by minimizing the finite horizon version of the cost function in (6).

THEOREM 2.1: Consider a continuous-time Markov chain evolving on $\mathcal{X}=\left\{e_{1}, \ldots, e_{n}\right\} \subset \mathbb{R}^{n}$, generated by (2). Let us define matrices $A=\sum_{i=1}^{m} \mu_{i, 0} G_{i}$ and $B_{i}=\sum_{j=1}^{m} \alpha_{i j} G_{j}$, where for all $1 \leq i, j \leq m, G_{i}$ and $\alpha_{i j}$ are introduced in (4) and (5), respectively. Assume that, for given $T \in \mathbb{R}_{>0}$ and $c:[0, T] \rightarrow \mathbb{R}^{n}$, differential equation

$\dot{k}(t)=-c(t)-A^{\top} k(t)+\frac{1}{4} \sum_{i=1}^{m}\left(S_{i}^{\top}+B_{i}^{\top} k(t)\right)^{.2} ; k(T)=k_{f}$,

has a solution on $[0, T]$ such that, for each $(t, x) \in[0, T] \times \mathcal{X}$, the operator $A-\sum_{i=1}^{m} \frac{1}{2}\left(k(t)^{\top} B_{i}+S_{i}\right) x B_{i}$ is an infinitesimal generator (see [6, pp. 124]). Then, the control law

$$
u_{i}(t, x)=-\frac{1}{2}\left(k(t)^{\top} B_{i}+S_{i}\right) x(t),
$$

for all $1 \leq i \leq m$, minimizes

$$
\begin{aligned}
J=\mathbb{E}\left\{\frac{1}{T} \int_{0}^{T} c(t)^{\top}\right. & x(t)+u(t)^{\top} S x(t) \\
& \left.+u(t)^{\top} u(t) \mathrm{d} t+\frac{1}{T} k_{f}^{\top} x(T)\right\} .
\end{aligned}
$$

PROOF: The proof of this theorem follows the same reasoning as in [3] to compute the optimal control law. See [7] for the detailed proof.

We can use Theorem 2.1 to calculate the infinite-horizon optimal policy through some algebraic manipulations.

COROLlary 2.2: Consider a continuous-time Markov chain evolving on $\mathcal{X}=\left\{e_{1}, \ldots, e_{n}\right\} \subset \mathbb{R}^{n}$, generated by (2). Let us define matrices $A=\sum_{i=1}^{m} \mu_{i, 0} G_{i}$ and $B_{i}=$ $\sum_{j=1}^{m} \alpha_{i j} G_{j}$, where for all $1 \leq i, j \leq m, G_{i}$ and $\alpha_{i j}$ are introduced in (4) and (5), respectively. Assume that, for a given $c \in \mathbb{R}^{n}$, nonlinear equation

$$
\left[\begin{array}{cc}
A^{\top} & -\mathbf{1} \\
\mathbf{1}^{\top} & 0
\end{array}\right]\left[\begin{array}{c}
k_{0} \\
\alpha
\end{array}\right]-\frac{1}{4}\left[\begin{array}{c}
\sum_{i=1}^{m}\left(S_{i}^{\top}+B_{i}^{\top} k_{0}\right)^{\cdot 2} \\
0
\end{array}\right]=\left[\begin{array}{c}
-c \\
0
\end{array}\right],
$$

has a solution such that, for all $x \in \mathcal{X}$, the operator $A-$ $\sum_{i=1}^{m} \frac{1}{2}\left(k_{0}^{\top} B_{i}+S_{i}\right) x B_{i}$ is an infinitesimal generator. Then, the control law

$$
u_{i}(t, x)=-\frac{1}{2}\left(k_{0}^{\top} B_{i}+S_{i}\right) x(t)
$$

for all $1 \leq i \leq m$, minimizes

$J=\lim _{T \rightarrow \infty} \mathbb{E}\left\{\frac{1}{T} \int_{0}^{T} c^{\top} x(t)+u(t)^{\top} S x(t)+u(t)^{\top} u(t) \mathrm{d} t\right\}$.

PROOF: First, noted that since $x(t) \in \mathcal{X}$ is always a bounded vector (i.e., $\|x(t)\|_{2}=1$ for all $t \geq 0$ ), we have the identity in (8) for any $k_{f} \in \mathbb{R}^{n}$. Therefore, without loss of generality, we can assume that $k_{f}=k_{0}$. According to Theorem 2.1, for any given $T>0$, we need to solve

$$
\dot{k}^{\prime}(t)=-c(t)-A^{\top} k^{\prime}(t)+\frac{1}{4} \sum_{i=1}^{m}\left(S_{i}^{\top}+B_{i}^{\top} k^{\prime}(t)\right)^{2},
$$

with the final condition $k^{\prime}(T)=k_{f}=k_{0}$ to get the optimal finite-horizon controller. Let us introduce the change of variable $k(t)=k^{\prime}(T-t)$ to transform (9) into

$\dot{k}(t)=c(t)+A^{\top} k(t)-\frac{1}{4} \sum_{i=1}^{m}\left(S_{i}^{\top}+B_{i}^{\top} k(t)\right)^{.2} ; k(0)=k_{0}$.

Defining $q(t)=k(t)-k_{0}-\alpha \mathbf{1} t$, we get

$$
\begin{aligned}
\dot{q}(t)= & \dot{k}(t)-\alpha \mathbf{1} \\
= & A^{\top} k(t)+c-\frac{1}{4} \sum_{i=1}^{m}\left(S_{i}^{\top}+B_{i}^{\top} k(t)\right)^{.2}-\alpha \mathbf{1} \\
= & A^{\top}\left(q(t)+k_{0}+\alpha \mathbf{1} t\right)+c-\alpha \mathbf{1} \\
& \quad-\frac{1}{4} \sum_{i=1}^{m}\left(S_{i}^{\top}+B_{i}^{\top}\left(q(t)+k_{0}+\alpha \mathbf{1} t\right)\right)^{\cdot 2} .
\end{aligned}
$$

Noting that $A^{\top} \mathbf{1}=0$ and $B_{i}^{\top} \mathbf{1}=0$ for all $1 \leq i \leq m$ [3], we get

$$
\begin{aligned}
& \dot{q}(t)=A^{\top}(q(t)+\left.k_{0}\right)+c-\alpha \mathbf{1} \\
&-\frac{1}{4} \sum_{i=1}^{m}\left(S_{i}^{\top}+B_{i}^{\top}\left(q(t)+k_{0}\right)\right)^{.2} \\
&=A^{\top} q(t)-\frac{1}{4} \sum_{i=1}^{m}\left(S_{i}^{\top}+B_{i}^{\top}\left(q(t)+k_{0}\right)\right)^{.2} \\
&+\frac{1}{4} \sum_{i=1}^{m}\left(S_{i}^{\top}+B_{i}^{\top} k_{0}\right)^{.2} .
\end{aligned}
$$

Noting that $q^{*}=0$ is an equilibrium of the nonlinear differential equation in (10), we realize that $q(t)=0$ for $t \in[0, T]$ since $q(0)=k(0)-k_{0}=0$. Thus, we get $k(T)=$ $k_{0}+\alpha \mathbf{1} T$, which results in $\left(k(T)^{\top} B_{i}+S_{i}\right)=\left(k_{0}^{\top} B_{i}+S_{i}\right)$, since $\mathbf{1}^{\top} B_{i}=0$ for all $1 \leq i \leq m$. As a result, the optimal controller is

$$
u_{i}(t, x)=-\frac{1}{2}\left(k_{0}^{\top} B_{i}+S_{i}\right) x(t),
$$

for all $1 \leq i \leq m$.

Corollary 2.2 introduces an optimal scheduling policy for allocating sampling instances among subsystems according to the cost function introduced in (6). By changing the design parameters $\xi_{\ell}$ for $1 \leq \ell \leq L$, we can tune the average sampling frequencies of the subsystems according to their required closed-loop performance. In addition, by adding an extra term to (6) whenever a new subsystem arrives or by 


$$
\lim _{T \rightarrow \infty} \mathbb{E}\left\{\frac{1}{T} \int_{0}^{T} c^{\top} x(t)+u(t)^{\top} S x(t)+u(t)^{\top} u(t) \mathrm{d} t\right\}=\lim _{T \rightarrow \infty} \mathbb{E}\left\{\frac{1}{T} \int_{0}^{T} c^{\top} x(t)+u(t)^{\top} S x(t)+u(t)^{\top} u(t) \mathrm{d} t+\frac{1}{T} k_{f}^{\top} x(T)\right\} .
$$

removing an old term from (6) whenever a subsystem leaves, we can accommodate for the changes in an ad-hoc networked system. In the remainder of this section, we analyze the asymptomatic statistical properties of the proposed stochastic scheduling policy.

\section{Effective Sampling Frequencies}

We use the notation $\left\{T_{i}^{\ell}\right\}_{i=0}^{\infty}$ to denote the sequence of time instances that subsystem $\ell$ is sampled. Mathematically, we define these time instances as

$$
T_{0}^{\ell}=\inf \left\{t \geq 0 \mid \exists \epsilon>0: x(t-\epsilon)=e_{n} \wedge x(t)=e_{\ell}\right\} .
$$

and

$$
T_{i+1}^{\ell}=\inf \left\{t \geq T_{i}^{\ell} \mid \exists \epsilon>0: x(t-\epsilon)=e_{n} \wedge x(t)=e_{\ell}\right\},
$$

for all $i \in \mathbb{Z}_{\geq 0}$. Furthermore, we define the sequence of random variables $\left\{\Delta_{i}^{\ell}\right\}_{i=0}^{\infty}$ such that $\Delta_{i}^{\ell}=T_{i+1}^{\ell}-T_{i}^{\ell}$ for all $i \in \mathbb{Z}_{\geq 0}$. These new random variables denote the time interval between any two successive sampling instances and play a crucial role in the closed-loop performance of the networked system. We also use the notation $M_{t}^{\ell}=$ $\max \left\{i \geq 1 \mid T_{i}^{\ell} \leq t\right\}$ to count the number of samples prior to any given time $t \in \mathbb{R}_{\geq 0}$. We make the following standing assumption:

Assumption 2.3: $\mathbb{P}\left\{T_{0}^{\ell}<\infty\right\}=1$ and $\mathbb{P}\left\{T_{1}^{\ell}<\infty\right\}=1$.

This assumption is without loss of generality since otherwise, subsystem $\ell$ would work open loop with a nonzero probability. Furthermore, this assumption is trivially satisfied if the number of subsystems is finite, the Markov chain is irreducible, and the rates of Poisson processes are finite and uniformly bounded away from zero.

LEMMA 2.4: $\left\{\Delta_{i}^{\ell}\right\}_{i=0}^{\infty}$ are independently and identically distributed random variables. Furthermore, if the sequence of sampling instances $\left\{T_{i}^{\ell}\right\}_{i=0}^{\infty}$ satisfy Assumption 2.3, then

$$
\lim _{t \rightarrow \infty} \frac{M_{t}^{\ell}}{t} \stackrel{\text { as }}{=} \frac{1}{\mathbb{E}\left\{\Delta_{i}^{\ell}\right\}},
$$

where $x \stackrel{a s}{=} y$ implies that $\mathbb{P}\{x=y\}=1$.

PROOF: The proof is a direct consequence of the Markov property and the law of large numbers (see [8]). See [7] for the detailed proof.

THEOREM 2.5: Let the sequence of sampling instances $\left\{T_{i}^{\ell}\right\}_{i=0}^{\infty}$ satisfy Assumption 2.3. Then, we have

$$
f_{\ell}=\lim _{T \rightarrow \infty} \mathbb{E}\left\{\frac{1}{T} \int_{0}^{T} e_{n}^{\top} x(t) \mathrm{d} N_{2 \ell}(t)\right\}=\frac{1}{\mathbb{E}\left\{\Delta_{i}^{\ell}\right\}} .
$$

PROOF: The proof is a direct consequence of using $M_{T}^{\ell}=$ $\int_{0}^{T} e_{n}^{\top} x(t) \mathrm{d} N_{2 \ell}(t)$ in Lemma 2.4 .

Let us introduce $p(t)=\mathbb{E}\{x(t)\}$. Following the same reasoning as in [3], we get

$$
\dot{p}(t)=A p(t)+\mathbb{E}\left\{\sum_{i=1}^{m} u_{i}(t, x(t)) B_{i} x(t)\right\}
$$

with the initial condition $p(0)=\mathbb{E}\{x(0)\}$. Substituting (7) inside (11), we get

$$
\begin{aligned}
\dot{p}(t) & =A p(t)-\frac{1}{2} \mathbb{E}\left\{\sum_{i=1}^{m}\left(k_{0}^{\top} B_{i}+S_{i}\right) x(t) B_{i} x(t)\right\} \\
& =A p(t)-\frac{1}{2} \mathbb{E}\left\{\sum_{i=1}^{m}\left(k_{0}^{\top} B_{i}+S_{i}\right)\left[\begin{array}{c}
x_{1}(t) \\
\vdots \\
x_{n}(t)
\end{array}\right] B_{i}\left[\begin{array}{c}
x_{1}(t) \\
\vdots \\
x_{n}(t)
\end{array}\right]\right\} \\
& =A p(t)-\frac{1}{2} \mathbb{E}\left\{\sum_{i=1}^{m} B_{i}\left[\begin{array}{c}
\left.x_{1}(t) \sum_{j=1}^{n}\left(k_{0}^{\top} B_{i}+S_{i}\right) e_{j} x_{j}(t)\right] \\
\vdots \\
x_{n}(t) \sum_{j=1}^{n}\left(k_{0}^{\top} B_{i}+S_{i}\right) e_{j} x_{j}(t)
\end{array}\right]\right\} .
\end{aligned}
$$

Note that $x_{h}(t) \sum_{j=1}^{n}\left(k_{0}^{\top} B_{i}+S_{i}\right) e_{j} x_{j}(t)=\left(k_{0}^{\top} B_{i}+\right.$ $\left.S_{i}\right) e_{h} x_{h}(t)$ since $x(t) \in \mathcal{X}$ (and elements of the set $\mathcal{X}$ are unit vectors in $\mathbb{R}^{n}$ ). Therefore, we get

$$
\begin{aligned}
\dot{p}(t) & =A p(t)-\frac{1}{2} \mathbb{E}\left\{\sum_{i=1}^{m} B_{i}\left[\begin{array}{c}
\left(k_{0}^{\top} B_{i}+S_{i}\right) e_{1} x_{1}(t) \\
\vdots \\
\left(k_{0}^{\top} B_{i}+S_{i}\right) e_{n} x_{n}(t)
\end{array}\right]\right\} \\
& =\left(A-\frac{1}{2} \sum_{i=1}^{m} B_{i} \operatorname{diag}\left(k_{0}^{\top} B_{i}+S_{i}\right)\right) p(t),
\end{aligned}
$$

where

$$
\operatorname{diag}\left(k_{0}^{\top} B_{i}+S_{i}\right)=\left[\begin{array}{ccc}
\left(k_{0}^{\top} B_{i}+S_{i}\right) e_{1} & \ldots & 0 \\
\vdots & \ddots & \vdots \\
0 & \ldots & \left(k_{0}^{\top} B_{i}+S_{i}\right) e_{n}
\end{array}\right] .
$$

Now, assuming that $p(t)$ converges exponentially to a nonzero value as time goes to infinity, that is, the ordinary differential equation in (12) is marginally stable (meaning all its eigenvalues except one that is on the origin have negative real parts), we can expand the expression for the effective sampling rates of the subsystems as (13). In (13), the third equality is a direct consequence of the fact that $x(t) \in \mathcal{X}$. We use this average sampling frequencies to bound the closed-loop performance of the networked system when our proposed optimal scheduling policy is implemented.

\section{Applications to Networked CONTROL}

In this section, we study networked control as an application of the proposed stochastic scheduling policy. Let us start by presenting the system model and controller. To simplify the proofs extensively, we first present the results for networked systems with impulsive controllers. However, later in Subsections III-C and III-D, we generalize these results to pulse and exponential controllers, respectively.

\section{A. System Model and Controller}

Consider the networked system illustrated in Figure 1, where subsystem $\ell$ for each $1 \leq \ell \leq L$, is a scalar stochastic system described by the Itô differential equation

$$
\mathrm{d} z_{\ell}(t)=\left(-\gamma_{\ell} z_{\ell}(t)+v_{\ell}(t)\right) \mathrm{d} t+\sigma_{\ell} \mathrm{d} w_{\ell}(t) ; z_{\ell}(0)=0,
$$




$$
\begin{aligned}
f_{\ell}=\lim _{T \rightarrow \infty} \mathbb{E}\left\{\frac{1}{T} \int_{0}^{T} e_{n}^{\top} x(t)\left(\mu_{2 \ell, 0}+\sum_{j=1}^{m} \alpha_{2 \ell, j} u_{j}\right) \mathrm{d} t\right\} & =\lim _{T \rightarrow \infty} \mathbb{E}\left\{\frac{1}{T} \int_{0}^{T} e_{n}^{\top} x(t)\left(\mu_{2 \ell, 0}-\frac{1}{2} \sum_{j=1}^{m} \alpha_{2 \ell, j}\left(k_{0}^{\top} B_{j}+S_{j}\right) x(t)\right) \mathrm{d} t\right\} \\
& =\lim _{T \rightarrow \infty} \frac{1}{T} \int_{0}^{T} e_{n}^{\top} p(t)\left(\mu_{2 \ell, 0}-\frac{1}{2} \sum_{j=1}^{m} \alpha_{2 \ell, j}\left(k_{0}^{\top} B_{j}+S_{j}\right) e_{n}\right) \mathrm{d} t \\
& =\left(\mu_{2 \ell, 0}-\frac{1}{2} \sum_{j=1}^{m} \alpha_{2 \ell, j}\left(k_{0}^{\top} B_{j}+S_{j}\right) e_{n}\right) e_{n}^{\top} \lim _{t \rightarrow \infty} p(t),
\end{aligned}
$$

with model parameters $\gamma_{\ell}, \sigma_{\ell} \in \mathbb{R}_{\geq 0}$. In (14), $v_{\ell}(t) \in \mathbb{R}$ denotes the control input of subsystem $\ell$. For each $1 \leq \ell \leq$ $L$, subcontroller $\ell$ receives noisy state measurements $\left\{y_{i}^{\ell}\right\}_{i=0}^{\infty}$ at time instances $\left\{T_{i}^{\ell}\right\}_{i=0}^{\infty}$, such that

$$
y_{i}^{\ell}=z_{\ell}\left(T_{i}^{\ell}\right)+n_{i}^{\ell} ; \quad \forall i \in \mathbb{Z}_{\geq 0},
$$

where $\left\{n_{i}^{\ell}\right\}_{i=0}^{\infty}$ denotes the measurement noise and is composed of independently and identically distributed Gaussian random variables with zero mean (i.e., $\mathbb{E}\left\{n_{i}^{\ell}\right\}=0$ ) and standard deviation $\eta_{\ell}$ (i.e., $\mathbb{E}\left\{\left(n_{i}^{\ell}\right)^{2}\right\}=\eta_{\ell}^{2}$ ). We assume that each subsystem is in feedback interconnection with a subcontroller governed by the control law

$$
v_{\ell}(t)=-\sum_{i=0}^{\infty} y_{i}^{\ell} f\left(t-T_{i}^{\ell}\right),
$$

where function $f: \mathbb{R} \rightarrow \mathbb{R} \cup\{\infty\}$ is chosen appropriately to yield a causal controller (i.e., $f(t)=0$ for all $t<0$ ). For instance, using $f(\cdot)=\delta(\cdot)$, where $\delta(\cdot)$ is an impulse function, results in an impulsive controller which simply resets the state of the subsystem to a neighborhood of the origin characterized by the amplitude of the measurement noise whenever a new measurement is received. In the next subsection, we start by analyzing impulsive controllers because it is relatively easier to bound their closed-loop performance explicitly. Other candidate functions $f(\cdot)$ are pulse and exponential functions. These functions can approximate the behavior of impulsive controllers in a more practical way.

\section{B. Performance Analysis: Impulsive Controllers}

In this subsection, we present an upper bound for the closed-loop performance of subsystems described in (14) when controlled by an impulsive controller. First, we need to prove the following simple lemma.

LEMMA 3.1: Let the function $g: \mathbb{R}_{\geq 0} \rightarrow \mathbb{R}$ be defined as $g(t)=c_{1} e^{-2 \gamma t}+\frac{c_{2}}{2 \gamma}\left(1-e^{-2 \gamma t}\right)$ with given scalars $c_{1}, c_{2} \in \mathbb{R}$ and $\gamma \in \mathbb{R}_{\geq 0}$ such that $2 \gamma c_{1} \leq c_{2}$. Then,

(a) $g$ is a non-decreasing function on its domain;

(b) $g$ is a concave function on its domain.

PROOF: For part (a), note that if $2 \gamma c_{1} \leq c_{2}$, the function $g(t)$ is continuously differentiable and $\mathrm{d} g(t) / \mathrm{d} t=-\left(2 \gamma c_{1}-\right.$ $\left.c_{2}\right) e^{-2 \gamma t} \geq 0$ for all $t \in \mathbb{R}_{\geq 0}$. Hence, $g(t)$ is a nondecreasing function on its domain (since it is continuous). On the other hand, for part (b), note that if $2 \gamma c_{1} \leq c_{2}$, the function $g(t)$ is double continuously differentiable and $\mathrm{d}^{2} g(t) / \mathrm{d} t^{2}=2 \gamma\left(2 \gamma c_{1}-c_{2}\right) e^{-2 \gamma t} \leq 0$ for all $t \in \mathbb{R}_{\geq 0}$. Therefore, $g(t)$ is a concave function on its domain.
When using impulsive controllers, the closed-loop subsystem $\ell$ is governed by

$$
\mathrm{d} z_{\ell}(t)=-\gamma_{\ell} z_{\ell}(t) \mathrm{d} t+\sigma_{\ell} \mathrm{d} w_{\ell}(t) ; \quad z_{\ell}\left(T_{i}^{\ell}\right)=-n_{i}^{\ell},
$$

for all $t \in\left[T_{i}^{\ell}, T_{i+1}^{\ell}\right)$. The next theorem presents an upper bound for the performance of this closed-loop system.

THEOREM 3.2: Assume that subsystem $\ell$ for $1 \leq \ell \leq$ $L$, is described by (14). Furthermore, let the sequence of sampling instances $\left\{T_{i}^{\ell}\right\}_{i=0}^{\infty}$ satisfy Assumption 2.3. Then, if $\eta_{\ell} \leq \sqrt{1 /\left(2 \gamma_{\ell}\right)} \sigma_{\ell}$, we get

$$
\mathbb{E}\left\{z_{\ell}^{2}(t)\right\} \leq \eta_{\ell}^{2} e^{-2 \gamma_{\ell} / f_{\ell}}+\frac{\sigma_{\ell}^{2}}{2 \gamma_{\ell}}\left(1-e^{-2 \gamma_{\ell} / f_{\ell}}\right) .
$$

otherwise, we get

$$
\mathbb{E}\left\{z_{\ell}^{2}(t)\right\} \leq \eta_{\ell}^{2}+\frac{\sigma_{\ell}^{2}}{2 \gamma_{\ell}}\left(1-e^{-2 \gamma_{\ell} / f_{\ell}}\right) .
$$

Proof: Using Itô's Lemma [6, p.49], we get

$$
\mathrm{d}\left(z_{\ell}^{2}(t)\right)=\left(-2 \gamma_{\ell} z_{\ell}^{2}(t)+\sigma_{\ell}^{2}\right) \mathrm{d} t+2 z_{\ell}(t) \sigma_{\ell} \mathrm{d} w_{\ell}(t) .
$$

for all $t \in\left[T_{i}^{\ell}, T_{i+1}^{\ell}\right)$. Therefore, we get

$$
\frac{\mathrm{d}}{\mathrm{d} t} \mathbb{E}\left\{z_{\ell}^{2}(t)\right\}=-2 \gamma_{\ell} \mathbb{E}\left\{z_{\ell}^{2}(t)\right\}+\sigma_{\ell}^{2}, \mathbb{E}\left\{z_{\ell}^{2}\left(T_{i}^{\ell}\right)\right\}=\eta_{\ell}^{2},
$$

which result in

$$
\mathbb{E}\left\{z_{\ell}^{2}(t) \mid \Delta_{i}^{\ell}\right\} \leq \eta_{\ell}^{2} e^{-2 \gamma_{\ell} t}+\frac{\sigma_{\ell}^{2}}{2 \gamma_{\ell}}\left(1-e^{-2 \gamma_{\ell} t}\right) .
$$

First, let us assume that $\eta_{\ell} \leq \sqrt{1 /\left(2 \gamma_{\ell}\right)} \sigma_{\ell}$. Then, we get

$$
\mathbb{E}\left\{z_{\ell}^{2}(t) \mid \Delta_{i}^{\ell}\right\} \leq \eta_{\ell}^{2} e^{-2 \gamma_{\ell} \Delta_{i}^{\ell}}+\frac{\sigma_{\ell}^{2}}{2 \gamma_{\ell}}\left(1-e^{-2 \gamma_{\ell} \Delta_{i}^{\ell}}\right),
$$

since according to Lemma 3.1 (a), when $\eta_{\ell} \leq \sqrt{1 /\left(2 \gamma_{\ell}\right)} \sigma_{\ell}$, the function $\eta_{\ell}^{2} e^{-2 \gamma_{\ell} t}+\sigma_{\ell}^{2} /\left(2 \gamma_{\ell}\right)\left(1-e^{-2 \gamma_{\ell} t}\right)$ is a nondecreasing function of time. Therefore, we get

$$
\begin{aligned}
\mathbb{E}\left\{z_{\ell}^{2}(t)\right\} & =\mathbb{E}\left\{\mathbb{E}\left\{z_{\ell}^{2}(t) \mid \Delta_{i}^{\ell}\right\}\right\} \\
& \leq \mathbb{E}\left\{\eta_{\ell}^{2} e^{-2 \gamma_{\ell} \Delta_{i}^{\ell}}+\frac{\sigma_{\ell}^{2}}{2 \gamma_{\ell}}\left(1-e^{-2 \gamma_{\ell} \Delta_{i}^{\ell}}\right)\right\} .
\end{aligned}
$$

Using Lemma 3.1 (b) and Jensen's inequality [6, p.320] shows that

$$
\begin{aligned}
& \mathbb{E}\left\{\eta_{\ell}^{2} e^{-2 \gamma_{\ell} \Delta_{i}^{\ell}}+\frac{\sigma_{\ell}^{2}}{2 \gamma_{\ell}}\left(1-e^{-2 \gamma_{\ell} \Delta_{i}^{\ell}}\right)\right\} \\
& \leq \eta_{\ell}^{2} e^{-2 \gamma_{\ell} \mathbb{E}\left\{\Delta_{i}^{\ell}\right\}}+\frac{\sigma_{\ell}^{2}}{2 \gamma_{\ell}}\left(1-e^{-2 \gamma_{\ell} \mathbb{E}\left\{\Delta_{i}^{\ell}\right\}}\right) .
\end{aligned}
$$

By substituting (20) into (19) while using Theorem 2.5, we get the inequality in (17). Now, for the case that $\eta_{\ell} \leq$ 
$\sqrt{1 /\left(2 \gamma_{\ell}\right)} \sigma_{\ell}$ is not satisfied, we can similarly derive the upper bound

$$
\mathbb{E}\left\{z_{\ell}^{2}(t) \mid \Delta_{i}^{\ell}\right\} \leq \eta_{\ell}^{2}+\frac{\sigma_{\ell}^{2}}{2 \gamma_{\ell}}\left(1-e^{-2 \gamma_{\ell} \Delta_{i}^{\ell}}\right),
$$

which results in (18) when using Lemma 3.1 (b) and Jensen's inequality [6, p.320]. This concludes the proof.

In the next two subsections, we generalize this result to two more practical controllers, namely, pulse and exponential controllers.

\section{Performance Analysis: Pulse Controllers}

In this subsection, we use a pulse function to approximate the impulse function behavior. Let us pick a constant $\rho \in$ $\mathbb{R}_{>0}$. For $t \in\left[T_{i}^{\ell}, T_{i+1}^{\ell}\right)$, we use the control law

$$
v_{\ell}(t)= \begin{cases}-y_{i}^{\ell} \gamma_{\ell} e^{-\gamma_{\ell} \rho} /\left(1-e^{-\gamma_{\ell} \rho}\right), & T_{i}^{\ell} \leq t \leq T_{i}^{\ell}+\rho, \\ 0, & T_{i}^{\ell}+\rho<t \leq T_{i+1}^{\ell},\end{cases}
$$

if $T_{i}^{\ell}+\rho \leq T_{i+1}^{\ell}$, and

$$
v_{\ell}(t)=-y_{i}^{\ell} \gamma_{\ell} e^{-\gamma_{\ell} \rho} /\left(1-e^{-\gamma_{\ell} \rho}\right), \quad T_{i}^{\ell} \leq t \leq T_{i+1}^{\ell},
$$

otherwise. This controller converges to the impulsive controller as $\rho$ tends to zero.

THEOREM 3.3: Assume that subsystem $\ell$ for $1 \leq \ell \leq$ $L$, is described by (14). Furthermore, let the sequence of sampling instances $\left\{T_{i}^{\ell}\right\}_{i=0}^{\infty}$ satisfy Assumption 2.3. Then, we get

$$
\mathbb{E}\left\{z_{\ell}^{2}(t)\right\} \leq \frac{\eta_{\ell}^{2}+\frac{\sigma_{\ell}^{2}}{2 \gamma_{\ell}}\left(1-e^{-2 \gamma_{\ell} / f_{\ell}}\right)}{1-\mathbb{P}\left\{T_{i+1}^{\ell}-T_{i}^{\ell}<\rho\right\}}
$$

PROOF: The proof follows the same reasoning as in the proof of Theorem 3.2. See [7] for the detailed proof.

Note that if $\rho$ tends to zero in (21), we would recover the same upper bound as in the case of the impulsive controller (18). This is true if $\lim _{\rho \rightarrow 0} \mathbb{P}\left\{T_{i+1}^{\ell}-T_{i}^{\ell}<\rho\right\}=$ 0 since the probability distribution of hitting-times of the underlying Markov chain is atom-less at the origin (which is satisfied when the Poisson jump rates are finite).

\section{Performance Analysis: Exponential Controllers}

In this subsection, we use an exponential function to approximate the impulse function behavior. Let us pick a constant $\theta \in \mathbb{R}_{>0} \backslash\left\{\gamma_{\ell}\right\}$. For all $t \in\left[T_{i}^{\ell}, T_{i+1}^{\ell}\right)$, we use the control law

$$
v_{\ell}(t)=\left(\gamma_{\ell}-\theta\right) y_{i}^{\ell} e^{-\theta\left(t-T_{i}^{\ell}\right)}
$$

This controller converges to the impulsive controller as $\theta$ approaches infinity.

THEOREM 3.4: Assume that subsystem $\ell$ for $1 \leq \ell \leq$ $L$, is described by (14). Furthermore, let the sequence of sampling instances $\left\{T_{i}^{\ell}\right\}_{i=0}^{\infty}$ satisfy Assumption 2.3. Then, we get

$$
\mathbb{E}\left\{z_{\ell}^{2}(t)\right\} \leq \frac{\eta_{\ell}^{2}+\frac{\sigma_{\ell}^{2}}{2 \gamma_{\ell}}\left(1-e^{-2 \gamma_{\ell} / f_{\ell}}\right)}{1-\mathbb{E}\left\{e^{-2 \theta\left(T_{i+1}^{\ell}-T_{i}^{\ell}\right)}\right\}}
$$

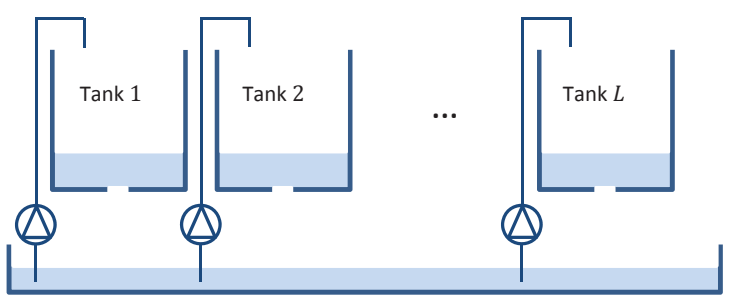

Fig. 3. An example of a networked system composed of several scalar decoupled subsystems.

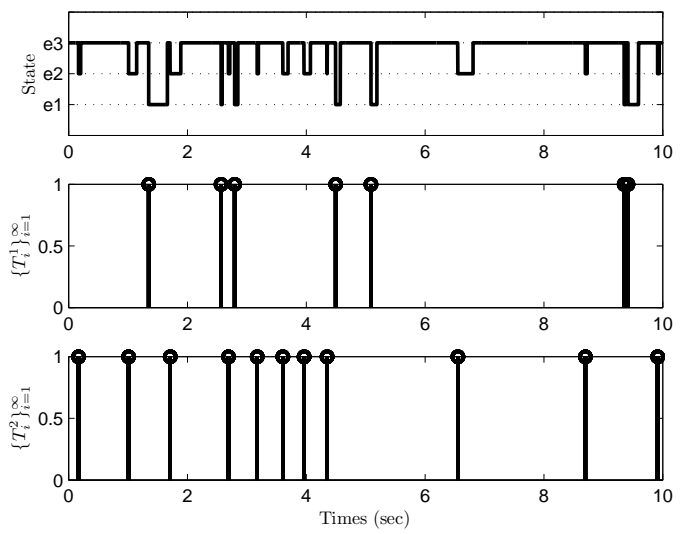

Fig. 4. An example of the state of the continuous-time Markov chain used in the optimal scheduling policy and its corresponding sampling instances for both subsystems.

PROOF: The proof follows the same reasoning as in the proof of Theorem 3.2. See [7] for the detailed proof.

Note that when $\theta$ tends to infinity, we would recover the same upper bound as in the case of the impulsive controller, since similarly $\lim _{\theta \rightarrow+\infty} \mathbb{E}\left\{e^{-2 \theta\left(T_{i+1}^{\ell}-T_{i}^{\ell}\right)}\right\}=0$ assuming that the probability distribution of hitting-times of the underlying Markov chain is atom-less at the origin.

\section{NumERicAl EXAMPLE}

In this section, we demonstrate the developed results on a networked system composed of $L$ decoupled water tanks illustrated in Figure 3, where each water tank can be modeled, around its stationary water level $h_{\ell}$, by

$\mathrm{d} z_{\ell}(t)=-\left(\frac{a_{\ell}}{A_{\ell}} \sqrt{\frac{g}{2 h_{\ell}}} z_{\ell}(t)+v_{\ell}(t)\right) \mathrm{d} t+\mathrm{d} w_{\ell}(t) ; z_{\ell}(0)=0$.

In this model, $A_{\ell}$ is the cross-section of water tank $\ell, a_{\ell}$ is the cross-section of its outlet hole, and $g$ is the acceleration of gravity. In addition, $z_{\ell}(t)$ and $v_{\ell}(t)$ denote the deviation of the tank's water level from its stationary point and its control input, respectively. The initial condition $z_{\ell}(0)=0$ shows that the tank's water level starts at its stationary level. However, due to factors such as input flow fluctuations, the water level drifts away from its stationary value.

We start by numerically demonstrating the developed results on a networked system composed of only two tanks (i.e., $L=2$ ). Let us fix parameters $A_{1}=A_{2}=1.00 \mathrm{~m}$, $a_{1}=0.20 \mathrm{~m}, a_{2}=0.10 \mathrm{~m}, g=9.80 \mathrm{~m} / \mathrm{s}^{2}, h_{1}=0.40 \mathrm{~m}$, and 

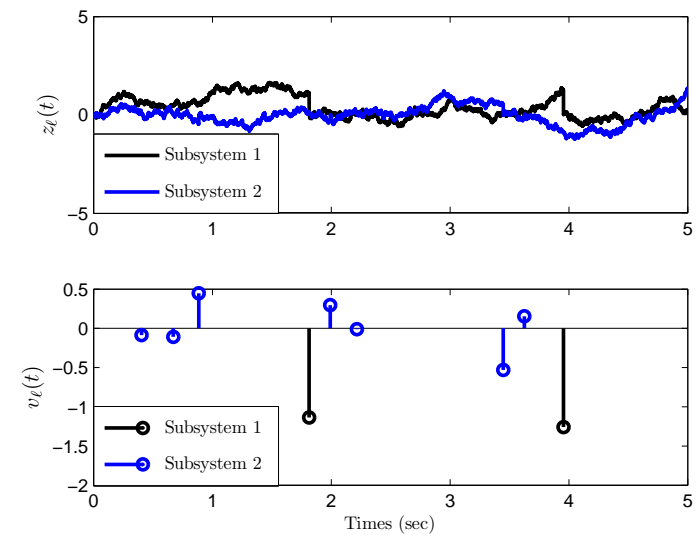

Fig. 5. An example of state and control of the closed-loop subsystems for the optimal scheduling policy when using impulsive controllers.
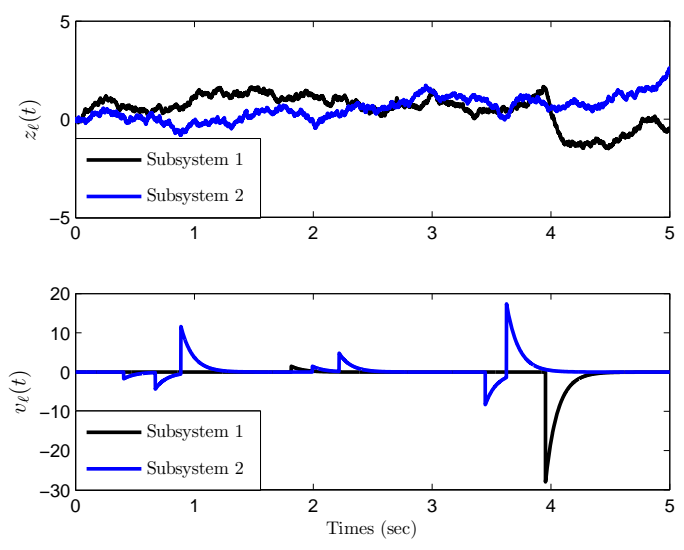

Fig. 6. An example of state and control of the closed-loop subsystems for the optimal scheduling policy when using exponential controllers.

$h_{2}=0.54 \mathrm{~m}$. For these physical parameter, the water tanks can be described by

$$
\begin{aligned}
& \mathrm{d} z_{1}(t)=\left(-0.7 z_{1}(t)+v_{1}(t)\right) \mathrm{d} t+\mathrm{d} w_{1}(t) ; z_{1}(0)=0, \\
& \mathrm{~d} z_{2}(t)=\left(-0.3 z_{2}(t)+v_{2}(t)\right) \mathrm{d} t+\mathrm{d} w_{2}(t) ; z_{2}(0)=0 .
\end{aligned}
$$

We sample these subsystems using the continuous-time Markov chain in (2) with $m=2 L=4$. We assume that $\mu_{i}(t)=\mu_{i, 0}+u_{i}(t)$ for $1 \leq i \leq 4$, where $\mu_{2 \ell, 0}=1$ and $\mu_{2 \ell-1,0}=10$ for $\ell=1,2$. We are interested in computing $u_{i}(t)$ for $1 \leq i \leq 4$, in order to minimize

$$
\begin{aligned}
J=\lim _{T \rightarrow \infty} \mathbb{E}\left\{\frac{1}{T}\right. & \int_{0}^{T} 0.5 e_{3}^{\top} x(t) \mathrm{d} N_{2} \\
& \left.+0.1 e_{3}^{\top} x(t) \mathrm{d} N_{4}+u(t)^{\top} u(t) \mathrm{d} t\right\} .
\end{aligned}
$$

Using Corollary 2.2, we get

$$
\left[\begin{array}{l}
u_{1}(t) \\
u_{2}(t) \\
u_{3}(t) \\
u_{4}(t)
\end{array}\right]=\left[\begin{array}{ccc}
-0.0228 & 0 & 0 \\
0 & 0 & -0.2272 \\
0 & -0.0228 & 0 \\
0 & 0 & -0.0272
\end{array}\right] x(t)
$$

Figure 4 illustrates an example of the continuous-time Markov chain state $x(t)$ and the sampling instances $\left\{T_{i}^{\ell}\right\}_{i=0}^{\infty}$ of both subsystems $\ell=1,2$. Using (13), we can calculate the
TABLE I

EXAMPLE OF AVERAGE SAMPLING FREQUENCIES

\begin{tabular}{|c|c||c|c|}
\hline$\xi_{1}$ & $\xi_{2}$ & $f_{1}$ & $f_{2}$ \\
\hline \hline 0.1 & 0.1 & 0.8040 & 0.8040 \\
0.5 & 0.1 & 0.6577 & 0.8279 \\
1.0 & 0.1 & 0.4656 & 0.8559 \\
2.0 & 0.1 & 0.0451 & 0.9045 \\
\hline
\end{tabular}

average sampling frequencies $f_{1}=0.6577$ and $f_{2}=0.8280$. We can tune these average sampling frequencies by changing the design parameters $\xi_{\ell}$ for $\ell=1,2$, in (6). Table I shows the average sampling frequencies of the subsystems calculated using (13) versus various choices of the design parameters $\xi_{\ell}$ for $\ell=1,2$. It is evident that when increasing (decreasing) $\xi_{\ell}$ for a given $\ell$, the average sampling frequency of subsystem $\ell$ decreases (increases).

Let us assume that each estimator has access to state measurements of its corresponding subsystem according to (15) with noise variance $\eta_{\ell}=0.3$ for $\ell=1,2$. Figures 5 and 6 illustrate the state and the control signal for both subsystems when using the impulsive and exponential controllers, respectively. For the exponential controller, we have fixed $\theta=10$. Note that in Figure 5, the control signal of the impulsive controller only portrays the energy that is injected to the subsystem (i.e., the integral of the impulse function) and not the exact value of the control input since by the definition of the impulse function, the actual value is infinite at sampling instances.

Figures 7 and 9 shows the closed-loop performance measure $\mathbb{E}\left\{z_{\ell}^{2}(t)\right\}$ approximated with 1000 simulations when using the impulsive and exponential controllers, respectively. The horizontal lines in Figure 7 show the theoretical upper bounds derived using Theorem 3.2; i.e., $\mathbb{E}\left\{z_{1}^{2}(t)\right\} \leq 0.6400$ and $\mathbb{E}\left\{z_{1}^{2}(t)\right\} \leq 0.9028$. As we expect from Section III, the closed-loop performance of the exponential controller is slightly worse than the closed-loop performance of the impulsive controller.

\section{A. Application to Ad-hoc Networked Systems}

Consider a networked system that can admit up to ten identical subsystems described by

$$
\mathrm{d} z_{\ell}(t)=\left(-0.3 z_{\ell}(t)+v_{\ell}(t)\right) \mathrm{d} t+\mathrm{d} w_{\ell}(t) ; z_{\ell}(0)=0,
$$

for $1 \leq \ell \leq L=10$. When all the subsystems are active, we sample these subsystems using the continuous-time Markov chain in (2) with $m=2 L=20$. We assume that $\mu_{i}(t)=$ $\mu_{i, 0}+u_{i}(t)$ for $1 \leq i \leq 20$, where $\mu_{2 \ell, 0}=1$ and $\mu_{2 \ell-1,0}=$ 5 for $1 \leq \ell \leq 10$. In this case, we are also interested in calculating an optimal scheduling policy that minimizes

$$
J=\lim _{T \rightarrow \infty} \mathbb{E}\left\{\frac{1}{T} \int_{0}^{T} \sum_{\ell=1}^{10} 0.1 e_{11}^{\top} x(t) \mathrm{d} N_{2 \ell}+u(t)^{\top} u(t) \mathrm{d} t\right\} .
$$

However, when some of the subsystems are inactive, we simply remove their corresponding nodes from the Markov chain flow diagram in Figure 2 and set their corresponding terms in (26) equal to zero. Let us assume that for $t \in[0,5)$, only two subsystems are active but for $t \in[5,10]$, all ten 


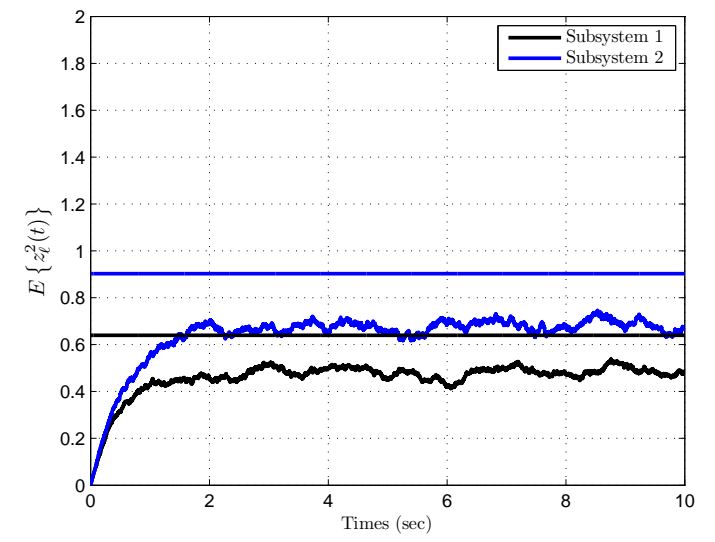

Fig. 7. Closed-loop performance $\mathbb{E}\left\{z_{\ell}^{2}(t)\right\}$ approximated with 1000 simulations for the optimal scheduling policy when using impulsive controllers.

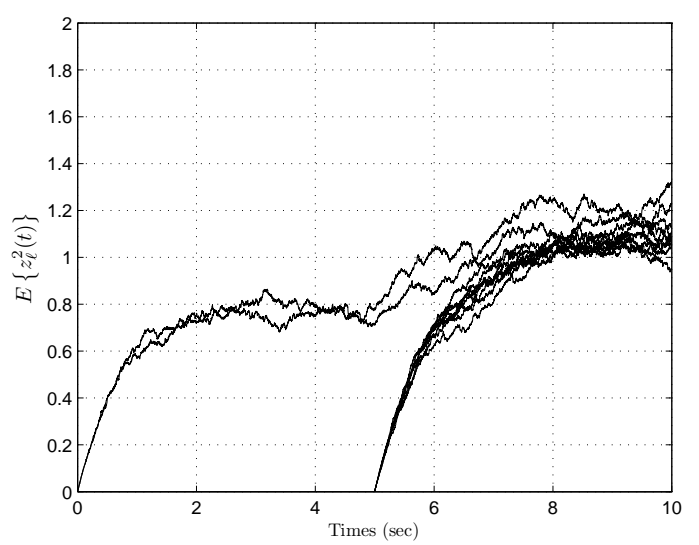

Fig. 8. Closed-loop performance $\mathbb{E}\left\{z_{\ell}^{2}(t)\right\}$ approximated with 1000 simulations when the number of subsystems increases from two to ten for the optimal scheduling policy.

subsystems are active. Let us assume that the subsystems are using impulsive controllers.

Figures 8 and 10 illustrate the closed-loop performance $\mathbb{E}\left\{z_{\ell}^{2}(t)\right\}$ approximated with 1000 simulations when using the optimal scheduling policy and the periodic scheduling policy, respectively. We set the sampling rates of periodic sampling policy exactly the same as the average sampling frequency of our optimal scheduling policy (when all the subsystems are active). Note that the saw-tooth behavior in this figure is due to the fact that for the periodic sampling policy, the sampling instances are fixed deterministically in advance to avoid transmission collision or interference. Since in the periodic scheduling policy, we have to fix the sampling instances in advance, we must determine the sampling periods according to the worst-case scenario (i.e., when the networked system is composed of ten subsystems). Hence, when using the periodic sampling, the networked system is not using its true potential for $t \in[0,5)$.

\section{CONCLusions}

We used a continuous-time Markov chain to optimally schedule the sampling instances of the subsystems in a networked system composed of several decoupled stable

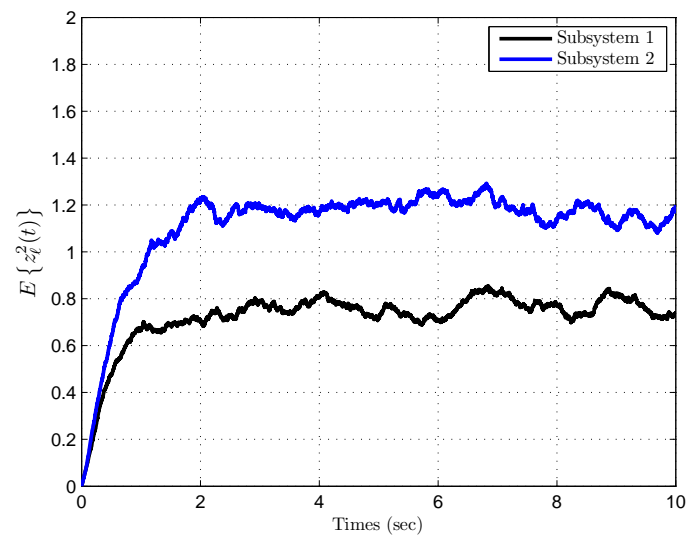

Fig. 9. Closed-loop performance $\mathbb{E}\left\{z_{\ell}^{2}(t)\right\}$ approximated with 1000 simulations for the optimal scheduling policy when using exponential controllers.

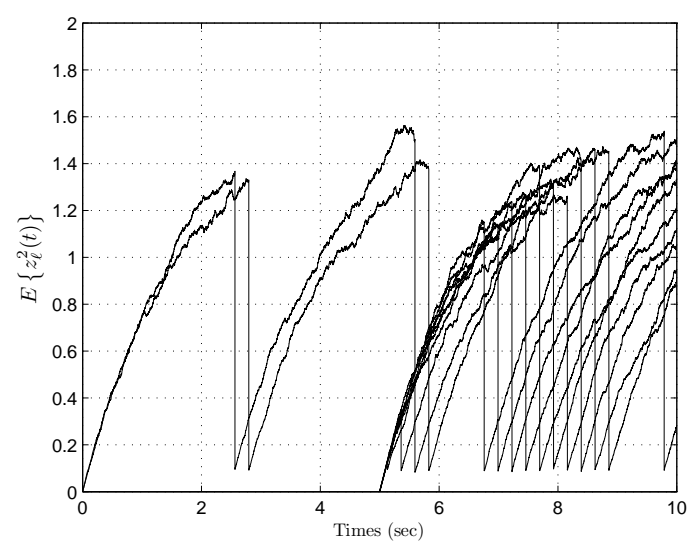

Fig. 10. Closed-loop performance $\mathbb{E}\left\{z_{\ell}^{2}(t)\right\}$ approximated with 1000 simulations when the number of subsystems increases from two to ten for the periodic scheduling policy.

scalar subsystems. We studied the statistical properties of this stochastic scheduling policy to compute bounds on the closed-loop performance of these networked system. As a future work, we could focus on extending the results to networked control of higher order subsystems or networked estimation.

\section{REFERENCES}

[1] K. J. Åström and B. Wittenmark, Computer controlled systems: theory and design. Prentice-Hall, 1984.

[2] G. F. Franklin, J. D. Powell, and M. L. Workman, Digital control of dynamic systems. Addison-Wesley, 1998.

[3] R. Brockett, "Optimal control of observable continuous time Markov chains," in Proceedings of the 47th IEEE Conference on Decision and Control, pp. $4269-4274,2008$.

[4] V. Gupta, T. Chung, B. Hassibi, and R. M. Murray, "On a stochastic sensor selection algorithm with applications in sensor scheduling and sensor coverage," Automatica, vol. 42, no. 2, pp. 251-260, 2006.

[5] R. Brockett, "Stochastic control." HYCON2-EECI Graduate School on Control Lecture notes, 2009. http://www.eeci-institute. eu/pdf/M015/RogersStochastic.pdf.

[6] B. K. Øksendal, Stochastic Differential Equations: An Introduction with Applications. Springer, 2003.

[7] F. Farokhi and K. H. Johansson, "Stochastic sensor scheduling with application to networked control," 2012. arXiv:1209.5180 [math.oc] http://arxiv.org/abs/1209.5180.

[8] R. G. Laha and V. K. Rohatgi, Probability theory. Wiley, 1979. 